\title{
Distribution of 'Candidatus Liberibacter asiaticus' Above and Below Ground in Texas Citrus
}

\author{
Eliezer S. Louzada, Omar Ed Vazquez, W. Evan Braswell, George Yanev, Madhavi Devanaboina, and Madhurababu Kunta
}

First, second, and sixth authors: Texas A\&M University-Kingsville Citrus Center, 312 N. International Blvd., Weslaco, TX 78599; third author: USDA APHIS PPQ CPHST, Mission Laboratory, Edinburg, TX 78541; and fourth and fifth authors: University of Texas Rio Grande Valley, Department of Mathematics, 1201 W. University Drive, Edinburg, TX 78539.

Accepted for publication 25 March 2016.

\begin{abstract}
Louzada, E. S., Vazquez, O. E., Braswell, W. E., Yanev, G., Devanaboina, M., and Kunta, M. 2016. Distribution of 'Candidatus Liberibacter asiaticus' above and below ground in Texas citrus. Phytopathology 106: 702-709.

Detection of 'Candidatus Liberibacter asiaticus' represents one of the most difficult, yet critical, steps of controlling Huanglongbing disease. Efficient detection relies on understanding the underlying distribution of bacteria within trees. To that end, we studied the distribution of ' $\mathrm{Ca}$. $\mathrm{L}$. asiaticus' in leaves of 'Rio Red' grapefruit trees and in roots of 'Valencia' sweet orange trees grafted onto sour orange rootstock. We performed two sets of leaf collection on grapefruit trees; the first a selective sampling targeting symptomatic leaves and their neighbors and the second a systematic collection disregarding symptomology. From uprooted orange trees, we exhaustively sampled fibrous roots. In this study, the presence of ' $\mathrm{Ca}$. L. asiaticus' was detected in leaves using real-time polymerase chain reaction (PCR) targeting the $16 \mathrm{~S}$ ribosomal gene and in roots using the $\mathrm{rpIJ} / \mathrm{rpIL}$ ribosomal protein genes and was confirmed with conventional PCR and sequencing of the rpIJ/rpIL gene in both tissues. Among

randomly collected leaves, 'Ca. L. asiaticus' was distributed in a patchy fashion. Detection of ' $\mathrm{Ca}$. L. asiaticus' varied with leaf symptomology with symptomatic leaves showing the highest frequency (74\%) followed by their neighboring asymptomatic leaves (30\%), while randomly distributed asymptomatic leaves had the lowest frequency (20\%). Among symptomatic leaves, we found statistically significant differences in mean number of bacterial cells with respect to both increasing distance of the leaf from the trunk and cardinal direction. The titer of ' $\mathrm{Ca}$. L. asiaticus' cells was significantly greater on the north side of trees than on the south and west sides. Moreover, these directions showed different spatial distributions of ' $\mathrm{Ca}$. L. asiaticus' with higher titers near the trunk on the south and west sides as opposed to further from the trunk on the north side. Similarly, we found spatial variation in ' $\mathrm{Ca}$. L. asiaticus' distribution among root samples. ' $\mathrm{Ca}$. L. asiaticus' was detected more frequently and bacterial abundances were higher among horizontally growing roots just under the soil surface $(96 \%)$ than among deeper vertically growing roots (78\%). Bacterial abundance declined slightly with distance from the trunk. These results point to paths of research that will likely prove useful to combating this devastating disease.
\end{abstract}

Huanglongbing (HLB), or citrus greening disease, is a threat affecting all citrus-producing regions worldwide. It is an aggressive, devastating, and incurable disease that cripples the citrus industry of affected areas. Even though the bacterium has been affecting the citriculture in Asia for more than a century (Bové 2006), it has reached the Americas only in the last 10 years. In the Americas, HLB was first reported in Brazil in 2004 (Coletta-Filho et al. 2004), was soon found in Florida in 2005 (Halbert 2005), and is currently widespread in Central America. In the United States, Florida is a perfect example of the devastating potential of HLB (Hodges and Spreen 2012), where in just 10 years, the whole industry has been affected and each year the decline of trees and production has shown that the potential for serious economic losses resulting in industry collapse is very high if a solution is not found.

HLB is believed to be caused by three related bacterial species of Liberibacter, of which 'Candidatus L. asiaticus' has caused the most widespread damage to the citrus industry worldwide. ' $\mathrm{Ca}$. L. asiaticus' is vectored by the Asian citrus psyllid (ACP) (Diaphorina citri Kuwayama) (Capoor et al. 1967; Martinez and Wallace 1967), which is well established in most of the commercial citrus production areas in the United States (Hall and Gottwald 2011).

Corresponding author: M. Kunta;

E-mail address: madhura.kunta@tamuk.edu

http://dx.doi.org/10.1094/PHYTO-01-16-0004-R

This article is in the public domain and not copyrightable. It may be freely reprinted with customary crediting of the source. The American Phytopathological Society, 2016.
Much remains unknown about this complex disease, highlighted by the inability to culture the putative disease agent. Additionally, the identification of infection is difficult, as it is believed that the lag between inoculation and symptom development can vary from six months to three years after natural inoculation (Bové 2006). Furthermore, the titers of ' $\mathrm{Ca}$. L. asiaticus' throughout the canopy vary so widely that only one shoot may test positive for ' $\mathrm{Ca}$. L. asiaticus' while the rest show negative results (Gottwald et al. 2008), suggesting a very patchy distribution in the canopy. Such variability within symptomatic trees necessitates visual inspection for disease symptoms to ensure bacterial detection (Kunta et al. 2014b).

Although natural inoculation occurs on leaves due to feeding behavior of ACP, recent studies show that ' $\mathrm{Ca}$. L. asiaticus' also is found among root tissue (Li et al. 2009), in which it appears to have a dramatic impact on root biomass (Johnson et al. 2014). As the loss of root biomass does not appear to be associated with phloem plugging, as in leaves (Johnson et al. 2014), the cause remains unknown.

In this research, we studied the distribution of ' $\mathrm{Ca}$. L. asiaticus' in leaves of grapefruit trees and in roots of sour orange rootstock grafted with 'Valencia' sweet orange. For grapefruit, our main interest was to determine if the patchy ' $\mathrm{Ca}$. L. asiaticus' distribution suggested elsewhere for sweet orange is also observed in grapefruit and to assess whether patterns of distribution exist that could aid detection. We sampled symptomatic leaves to determine variation in ' $C a$. L. asiaticus' concentrations with respect to distance and direction from the trunk. To confirm patchy ' $\mathrm{Ca}$. L. asiaticus' distribution, a second systematic collection of symptomatic and asymptomatic leaves was performed from one branch in each quadrant at regular intervals from the trunk. 
Most of the studies on ' $\mathrm{Ca}$. L. asiaticus' distribution have been performed in the canopy of the trees. However, recent reports indicate that roots are among the first tissues to show disease symptoms (Johnson et al. 2014). The distribution of ' $C a$. L. asiaticus' in the above-ground parts of HLB-infected trees has been suggested to be uneven with asymptomatic leaves in symptomatic trees producing negative real-time PCR test results for the presence of ' $C a$. L. asiaticus' (Kunta et al. 2014a and b). The results reported were for random sampling of asymptomatic leaves in symptomatic 'Valencia' sweet orange trees, but a detailed distribution study was not performed. Considering that more than $70 \%$ of the Texas citrus industry is planted with grapefruit and that detailed ' $C a$. L. asiaticus' distribution in grapefruit canopy under Texas conditions has never been reported, we decided to undertake this study. Here, we report a detailed study on horizontal and vertical distribution of ' $\mathrm{Ca}$. L. asiaticus' in fibrous roots of sour orange rootstock. The grapefruit and sweet orange trees that were sampled in this study were located in San Juan, Texas, where HLB was first found in Texas in 2012 (da Graça et al. 2015). The results from this research should be very informative for development of sample collection methods for detection and management strategies for HLB.

\section{MATERIALS AND METHODS}

Selective leaf sampling (collection 1). Leaf tissue was collected in June 2014 from four HLB-symptomatic, field-grown naturally

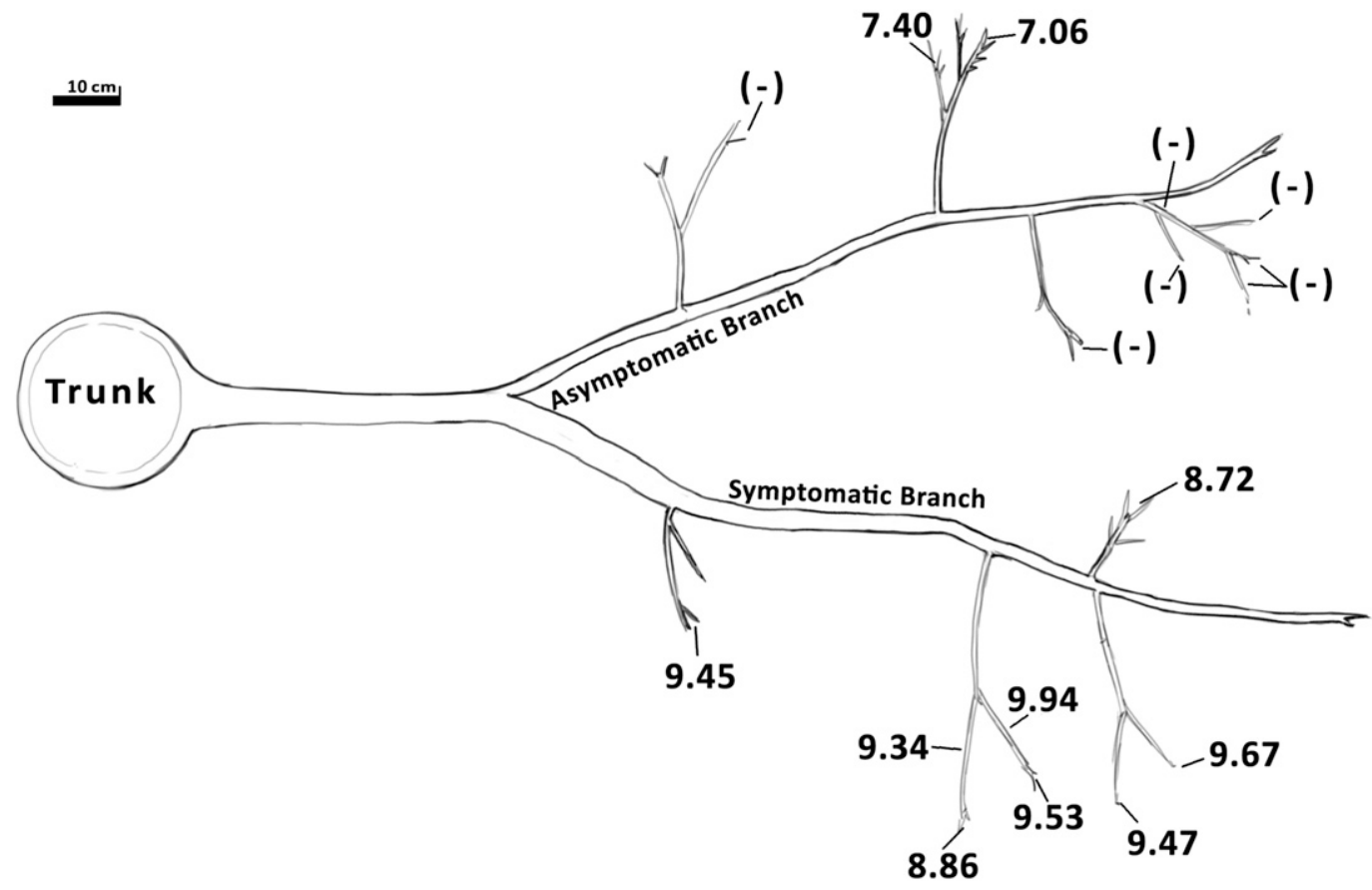

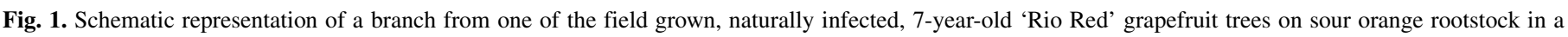

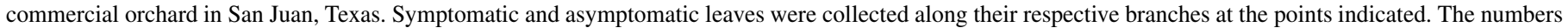
indicate the log of 'Candidatus Liberibacter asiaticus' genome equivalents per gram of tissue.

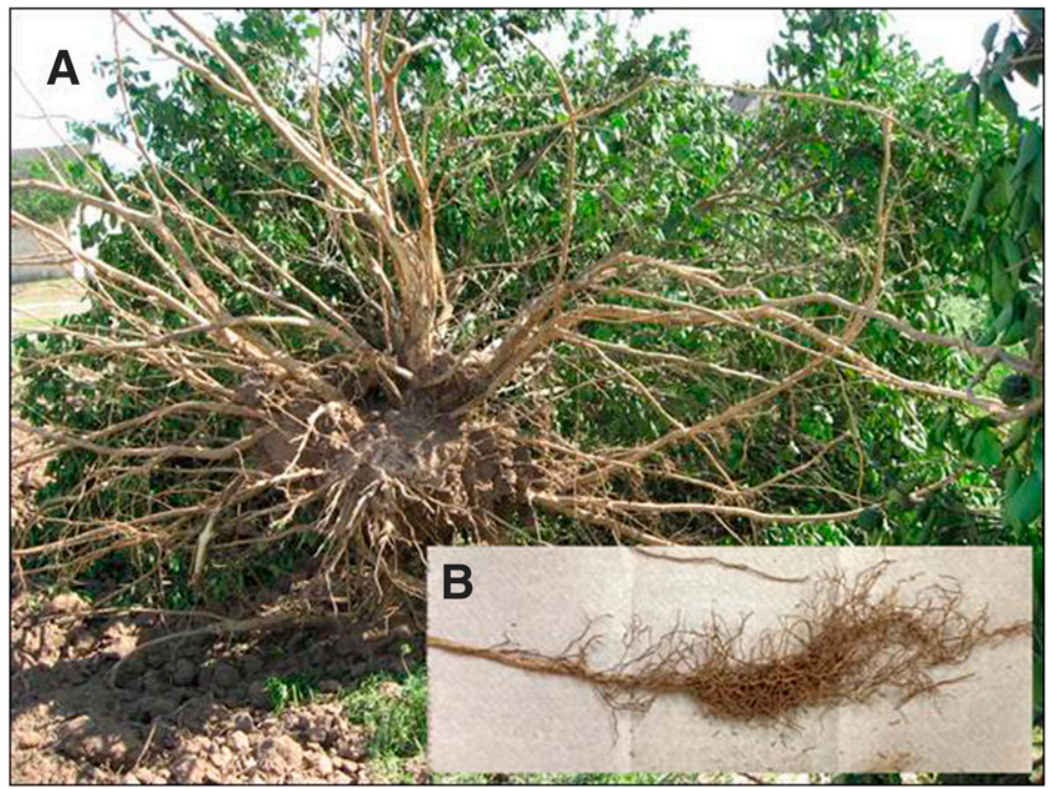

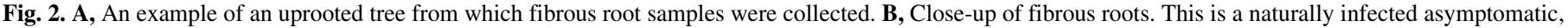
7-year-old 'Valencia' sweet orange tree on sour orange rootstock grown in a commercial orchard in San Juan, Texas. 
infected, 7-year-old 'Rio Red' grapefruit trees on sour orange rootstock in San Juan, TX. The 'Rio Red' grapefruit trees used were located on the southwestern-most block of the orchard. These trees were detected as HLB positive in January 2012. However, considering the large number of trees in the orchard infected and long latency periods reported for HLB, they indeed seem to be infected at least three years earlier. One branch showing clear symptoms of HLB was selected from each cardinal direction (north, south, east, west) around each tree and symptomatic leaves were sampled all along the selected branch, while recording the total distance from the trunk to the leaf (Fig. 1). From each of four trees, when the branch would split and asymptomatic leaves were present, these leaves were collected.

Systematic leaf sampling (collection 2). In March 2015, to study the patchy distribution of ' $\mathrm{Ca}$. L. asiaticus' throughout the canopy, a total of 72 additional leaf samples were collected (symptomatic or asymptomatic) every $25 \mathrm{~cm}$ from the trunk off one randomly chosen branch in each quadrant of the canopy of three trees out of the four trees described above. The leaves were categorized into HLB symptomatic, HLB asymptomatic, and leaves with atypical HLB symptoms.

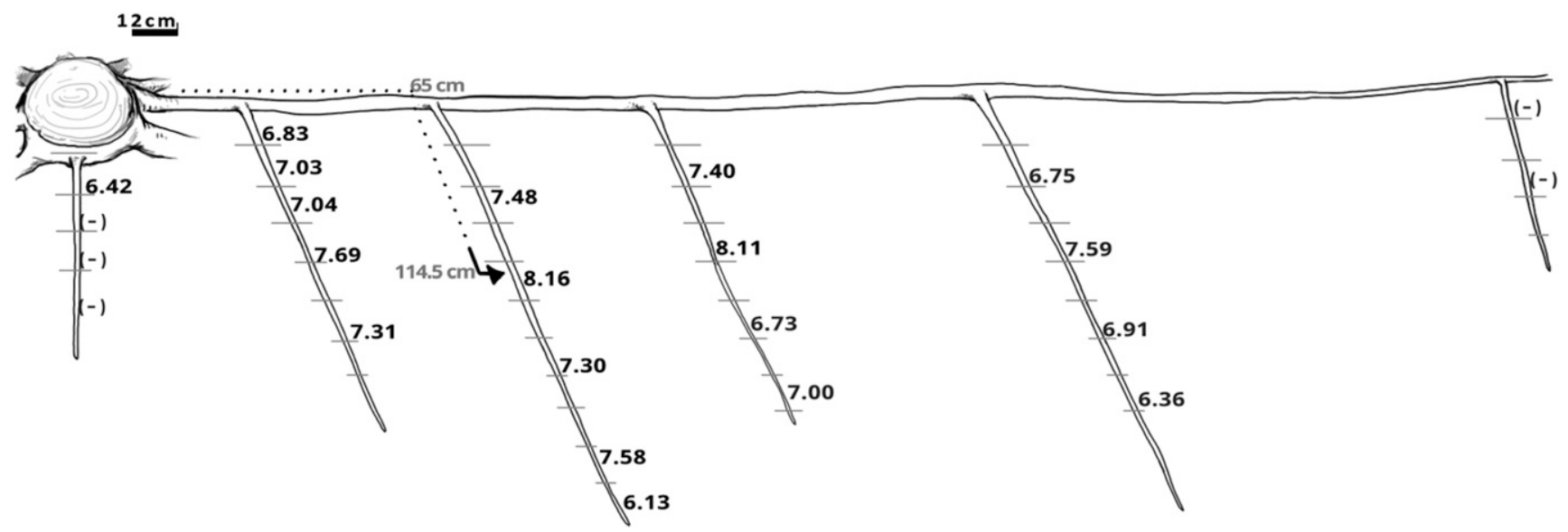

Fig. 3. Schematic representation of root sample collection from a horizontally growing root of one of the asymptomatic, 7-year-old 'Valencia' sweet orange trees on sour orange rootstock grown in a commercial orchard in San Juan, TX. Hash marks indicate sample locations from which fibrous roots were collected. Numbers indicate the $\log$ of 'Candidatus Liberibacter asiaticus' genome equivalents per gram of tissue, while - indicates that ' $C a$. L. asiaticus' was not detected by real-time polymerase chain reaction. The dotted line indicates the distance from the trunk to a sample collection point.

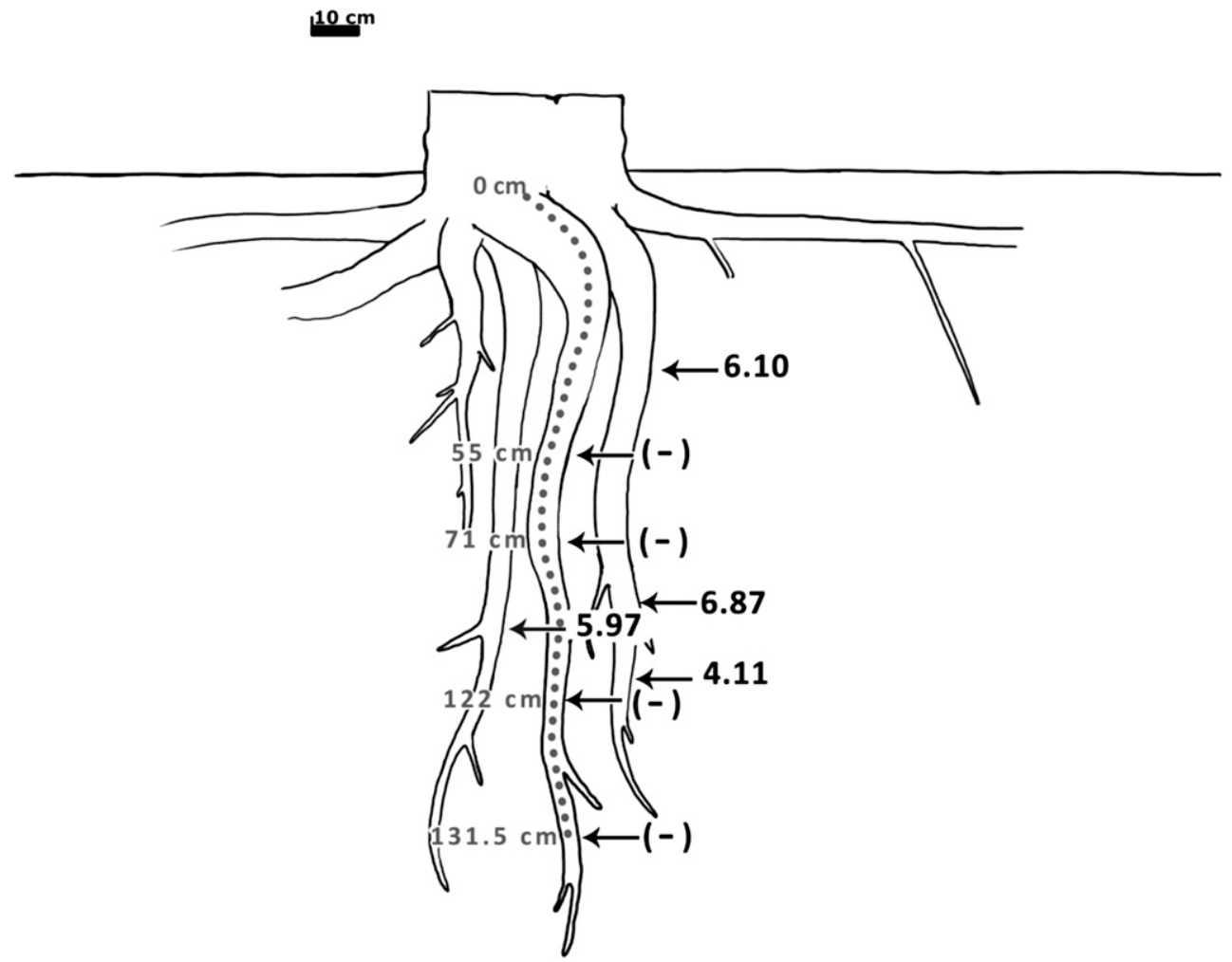

Fig. 4. Schematic representation of root sample collection from a vertically growing root of one of the asymptomatic, 7-year-old 'Valencia' sweet orange trees on sour orange rootstock grown in a commercial orchard in San Juan, TX. Arrows indicate locations from which fibrous roots were collected. Numbers indicate the $\log$ of 'Candidatus Liberibacter asiaticus' genome equivalents per gram of tissue, while - indicates that ' $\mathrm{Ca}$. L. asiaticus' was not detected by real-time polymerase chain reaction. The dotted line indicates the distance from the trunk to a sample collection point. 
Root sampling. Root tissue was collected in August 2013 from five naturally infected, asymptomatic, 7-year-old 'Valencia' sweet orange trees grafted on sour orange rootstock grown in a commercial orchard in San Juan, Texas (Fig. 2). These trees were sampled in a previous study three months prior, and all were found to be asymptomatic. Real-time PCR analysis of leaves showed that each of these trees was ' $\mathrm{Ca}$. L. asiaticus' negative; however, in the roots, all but one was ' $\mathrm{Ca}$. L. asiaticus' positive (Kunta et al. 2014b). In this study, we uprooted these trees and fibrous roots were exhaustively sampled from horizontally growing roots up to 3.6 meters from the trunk (Fig. 3) and from vertically growing roots down to 1.5 meters from the trunk (Fig. 4). The distance was measured along the length of the branch or root from the trunk to the sample collection point.

DNA extraction from leaves. Total DNA was isolated from individual leaves using the Qiagen DNeasy plant mini kit (Qiagen, Hilden, Germany). Midrib and petiole were separated from the leaf and were chopped. Fifty milligrams of chopped midrib and petiole was homogenized in a 2-ml lysing matrix A tube (MP Biomedicals, Santa Ana, CA) with buffer AP1, RNase A, plus two 4.0-mm zirconia beads and was pulverized for $3 \mathrm{~min}$ at a shaking speed of 2,100 oscillations per minute, using a Mini-Beadbeater-96 (BioSpec Products, Inc., Bartlesville, OK). Total DNA was eluted in $100 \mu \mathrm{l}$ of nuclease-free water.

DNA extraction from roots. Root tissue samples were stored in paper bags and were dried at $60^{\circ} \mathrm{C}$ for $1 \mathrm{~h}$. Any excess dirt was shaken off the roots and they were chopped with a razor blade. Fifty milligrams of root tissue plus two 4.0-mm zirconia beads (BioSpec Products, Inc.) were placed in a 2-ml screw cap with and O-ring microcentrifuge tube and were pulverized for $3 \mathrm{~min}$ at a shaking speed of 2,100 oscillations per minute in a Mini-Beadbeater-96. The extraction buffer was added, the samples were pulverized once more for $3 \mathrm{~min}$ at the same speed, and DNA was extracted with the PowerPlant Prohtp 96 well DNA isolation kit (MO Bio Laboratories, Carlsbad, CA).

Real-time PCR. For detecting ' $\mathrm{Ca}$. L. asiaticus' in leaves, realtime PCR assays were performed, using the HLBaspr primer and probe set, which targets the $16 \mathrm{~s}$ rDNA of ' $\mathrm{Ca}$. L. asiaticus' (Li et al. 2006). Previous data have shown that the HLBaspr primer and probe system produce nonspecific amplification from citrus root tissue from Texas (Kunta et al. 2014b). Therefore, to detect ' $\mathrm{Ca}$. L. asiaticus' in the root tissue, real-time PCR assays were performed using the species-specific CQULA primer and probe set that targets the $\mathrm{rpIJ} / \mathrm{rpIL}$ ribosomal protein gene (Wang et al. 2006). Each realtime PCR was replicated three times for each DNA extract. For each extraction, one real-time PCR targeting plant DNA was performed separately to ensure successful DNA extraction (Li et al. 2006).

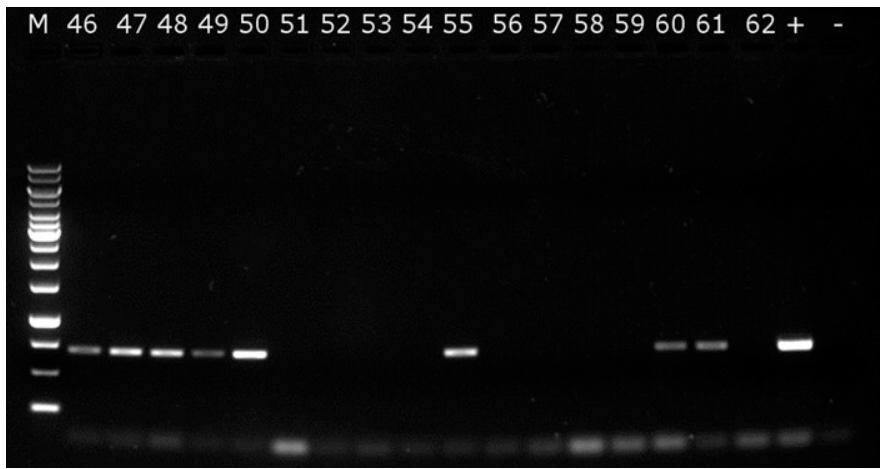

Fig. 5. The specific conventional polymerase chain reaction (PCR) amplification products with a size of $703 \mathrm{bp}$, obtained using A2/J5 primers on the DNA extract of Huanglongbing-symptomatic 'Rio Red' grapefruit leaves. $\mathrm{M}=$ Thermo Scientific Gene Ruler 1-kb DNA ladder, lanes 46 to $62=$ symptomatic leaves, $+=$ 'Ca . L. asiaticus' PCR positive control DNA, and $-=$ nontemplate water control.
All real-time PCR assays were performed using $2 \mu \mathrm{l}$ of total DNA extract in a $25-\mu$ l reaction mixture with $2.5 \mu \mathrm{l} 10 \times$ PCR buffer (Invitrogen, Carlsbad, CA), $2.5 \mathrm{mM} \mathrm{MgCl} 2,0.2 \mathrm{mM}$ each dNTP, $0.2 \mu \mathrm{M}$ each primer, and 1 Unit platinum Taq DNA polymerase (Invitrogen) in a CFX96 Touch real-time PCR detection system (Bio-Rad Laboratories, Hercules, CA). The threshold cycle (Ct) values obtained from the assays were used to determine the presence of the target sequence in the samples. Although the real-time PCR targets differ between leaf and root assays, we treated the data identically and conservatively; $C_{t}$ values of 37 or greater were considered negative for containing the target sequence ( $\mathrm{Li}$ et al. 2007, 2008). Using the models described by Li et al. (2008) $(y=18.697-$ $0.3432 x)$ for leaf samples and Wang et al. (2006) $(y=13.82-$ $0.2866 x$ ) for root samples, the $C_{t}$ values obtained were converted into ' $\mathrm{Ca}$. L. asiaticus' genome equivalent counts (cell counts) for use in all statistical analyses.

Conventional (c)PCR. The real-time PCR results for all leaf and root samples were further verified by $\mathrm{CPCR}$, using $\mathrm{A} 2 / \mathrm{J} 5$ primers. The $\mathrm{A} 2 / \mathrm{J} 5$ primer set amplifies the ribosomal protein genes of the $\beta$ operon in ' $\mathrm{Ca}$. L. asiaticus' (Hocquellet et al. 1999). The conventional PCR amplicon for each extract was visualized by agarose gel electrophoresis and was stained with ethidium bromide. The PCR amplicons were purified with the Qiaquick gel extraction kit (Qiagen). The purified PCR products were cloned using the TOPO TA cloning kit for sequencing (Life Technologies, Carlsbad, CA) and were sequenced with the universal M13 reverse and T7 promotor primers in order to verify the amplification products from cPCR. The sequences obtained were visually inspected, edited, and
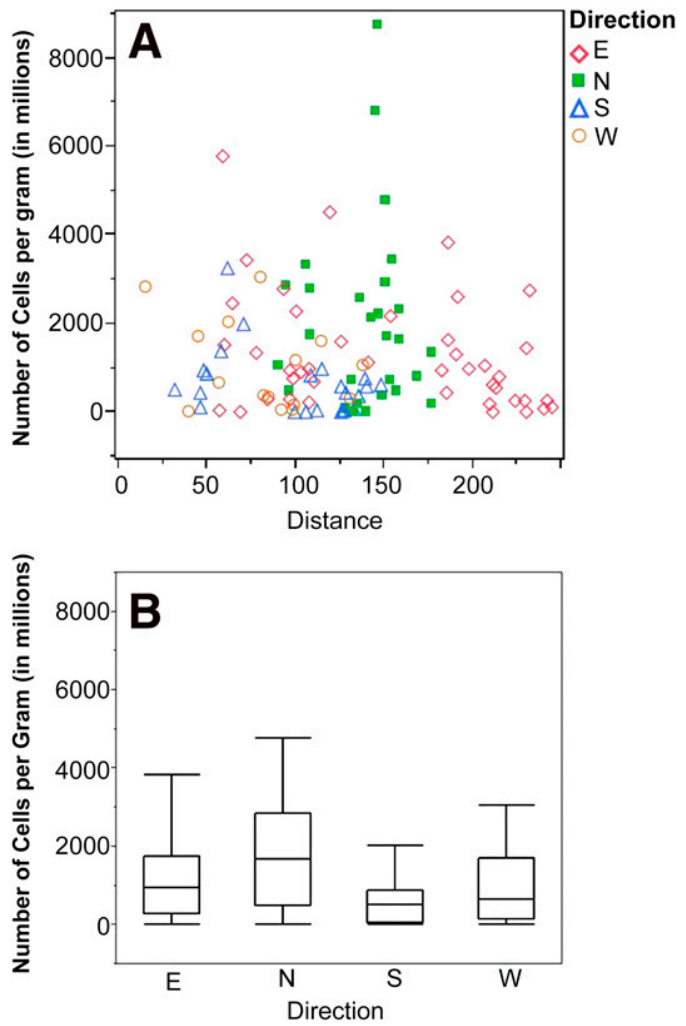

Fig. 6. The number (in millions) of 'Candidatus Liberibacter asiaticus' genome equivalents per gram of leaf tissue estimated in symptomatic leaves collected from 'Rio Red' grapefruit trees. A, Scatterplot of estimated 'Candidatus Liberibacter asiaticus' genome equivalents present in the DNA extracts by distance (in $\mathrm{cm}$ ) for different directions. B, Box plot for number of ' $\mathrm{Ca}$. L. asiaticus' genome equivalents per gram of tissue by direction. The center line represents the median, the outer lines represent the first and third quartiles, and the whiskers encapsulate all the data points that are not outliers (beyond $1.5 \times$ the interquartile range). 
subjected to BLASTn (Altschul et al. 1990) analysis against GenBank for homology and specific identification of the sequences.

Statistical analysis. Covariance modeling (ANCOVA) was undertaken to test the significance of independent variables and their interactions on the number of bacterial cells per gram of plant tissue. The response variable was measured as the average number of bacterial cells estimated from three real-time PCRs per DNA extract. ANCOVA and Tukey's highly significant difference (HSD) test for means separation were performed, using JMP 11.0.0 (2013), SAS Institute Inc. (Cary, NC) and a type I error probability of 5\% to determine statistical significance.

Canopy data analysis. Two independent variables, distance and direction, along with their interaction were used to predict the number of ' $\mathrm{Ca}$. L. asiaticus' cells per gram of leaf tissue. Distance was measured in centimeters along branch points from trunk to sample location, while direction was categorized as the cardinal direction class from which the sample was taken. The initial sample size of 133 leaves (collection 1) was right-censured at real-time PCR $C_{t} \geq 37$, bringing the effective sample size to 108 .

Root data analysis. Three independent variables, root type, distance, and individual tree, were used to predict the logarithm-transformed number of ' $\mathrm{Ca}$. L. asiaticus' cells per gram of root tissue. Root type was categorized as horizontal or vertical based on the direction of growth, distance was measured as in the canopy, and individual tree was included to identify individual tree-based effects. The initial sample size of 160 root samples were right-censured at real-time PCR $C_{t} \geq 37$, resulting in a sample size of 149 .

\section{RESULTS}

Detection of ' $\mathrm{Ca}$. L. asiaticus' in selectively collected leaves (collection 1). In total, 163 leaf samples (133 symptomatic and 30 asymptomatic) were collected from four 'Rio Red' grapefruit trees on sour orange rootstock. The symptomatic leaves showed typical symptoms of blotchy mottle and corky vein; the fruits were asymmetrical with aborted seeds and vascular browning, and tree twigs showed die-back on all sides. Real-time PCR detected the presence of ' $\mathrm{Ca}$. L. asiaticus' in 108 (66\%) of the total leaves. From the 133 symptomatic leaves, $74 \%$ were positive, while from the 30 asymptomatic leaves, $30 \%$ were positive. cPCR was able to detect 106 of all real-time PCR positive samples (98\% of the real-time PCR positives) (Fig. 5). Given the lower detection limit of

TABLE 1. Frequency of 'Candidatus Liberibacter asiaticus' detection by real-time polymerase chain reaction (PCR) and average real-time PCR threshold cycle $(\mathrm{Ct})$ of those detections among systematically collected leaves (at $25-\mathrm{cm}$ intervals from the trunk) of three infected and symptomatic 'Rio Red' grapefruit trees ${ }^{\mathrm{a}}$

\begin{tabular}{|c|c|c|c|c|c|c|c|c|}
\hline \multirow[b]{2}{*}{ Tree } & \multicolumn{2}{|c|}{ All leaves } & \multicolumn{2}{|c|}{ Asymptomatic leaves } & \multicolumn{2}{|c|}{ Symptomatic leaves } & \multicolumn{2}{|c|}{ Atypical symptoms } \\
\hline & CLas+/total & Average $\mathrm{Ct}$ & CLas+/total & Average $\mathrm{Ct}$ & CLas+/total & Average $\mathrm{Ct}$ & CLas+/total & Average $\mathrm{Ct}$ \\
\hline 1 & $4 / 21(19 \%)$ & 28.2 & $3 / 20(15 \%)$ & 29.4 & $1 / 1(100 \%)$ & 24.6 & - & - \\
\hline 2 & $10 / 28(35.7 \%)$ & 30.6 & $8 / 24(33 \%)$ & 31.4 & $2 / 2(100 \%)$ & 27.3 & $0 / 2(0 \%)$ & _- \\
\hline Total & $18 / 72(25 \%)$ & 30.3 & $13 / 65(20 \%)$ & 30.9 & $4 / 4(100 \%)$ & 27.6 & $1 / 3(33 \%)$ & \\
\hline
\end{tabular}

${ }^{a}$ CLas $+=$ Number of leaf samples that gave a positive real-time PCR reaction for the presence of 'Candidatus $\mathrm{L}$. asiaticus'; $\mathrm{Ct}=\mathrm{threshold} \mathrm{cycle} \mathrm{values} \mathrm{generated}$ in the real-time PCR reactions.

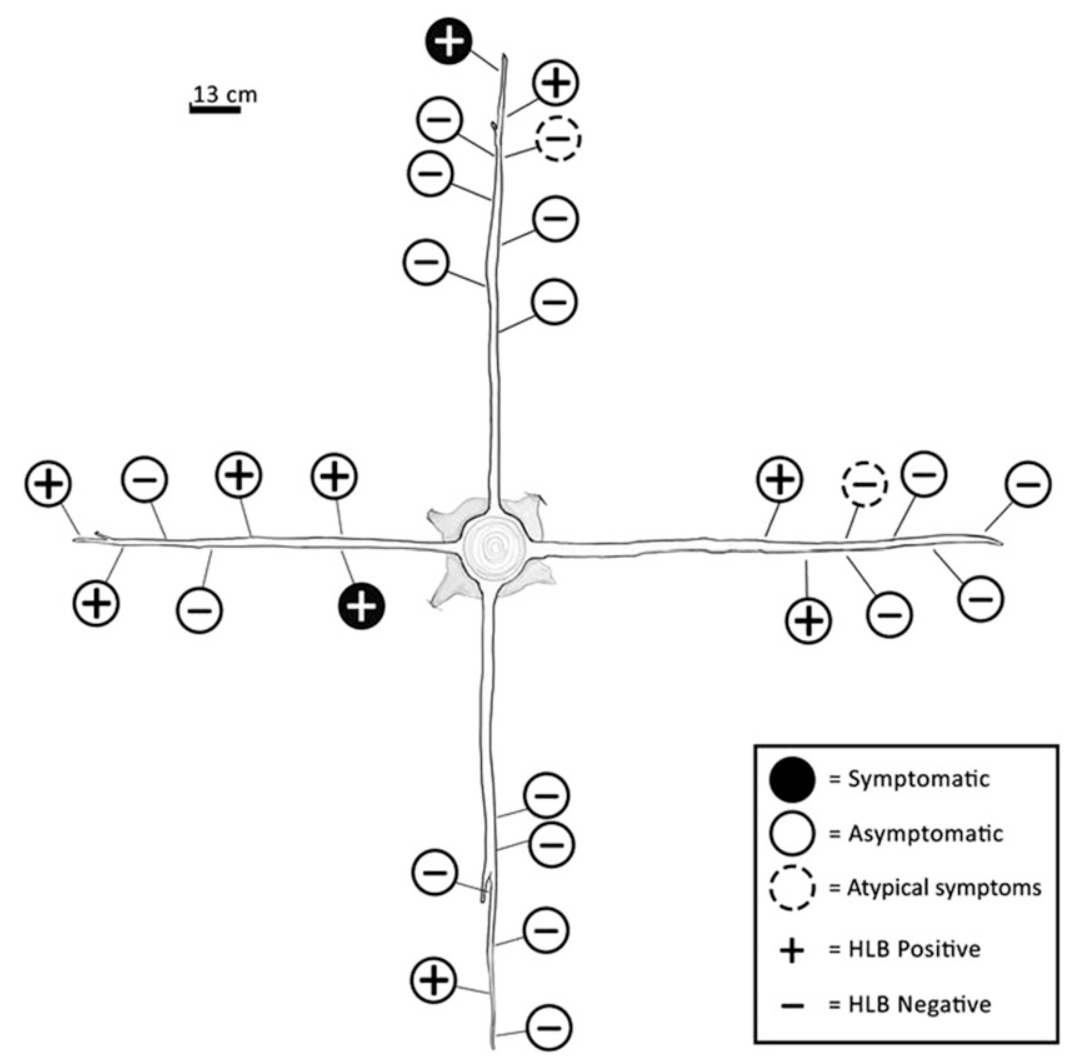

Fig. 7. Diagram depicting the locations of 'Candidatus Liberibacter asiaticus' positive (+) and negative (-) leaf samples collected at 25 -cm intervals from a representative 'Rio Red' grapefruit tree (tree number 2) trunk. The other trees exhibited similar patterns. The presence of ' $\mathrm{Ca}$. L. asiaticus' was detected by realtime polymerase chain reaction using HLBaspr primers and probe set. 
cPCR, we based our subsequent statistical analyses on the real-time PCR results. The positive leaf samples were located between 15 and $245 \mathrm{~cm}$ from the trunk. There was no significant variation among trees, so the individual leaf samples were pooled for further analysis. The ANCOVA test indicated overall statistically significant variation in mean number of bacterial cells with respect to distance $(P$ value 0.0313$)$ and direction $(P$ value 0.0013$)$. The Tukey HSD test indicated significantly greater ' $\mathrm{Ca}$. L. asiaticus' abundance to the north than in the south (Fig. 6B). In the north, where the number of ' $C a$. L. asiaticus' cells detected was significantly higher than in the other directions, the peak abundance was at $150 \mathrm{~cm}$, while it was between 50 and $75 \mathrm{~cm}$ for all other directions. Moreover, the distribution of ' $\mathrm{Ca}$. L. asiaticus' was also most uniform in the eastern branches (Fig. 6A).

Detection of ' $\mathrm{Ca}$. $\mathrm{L}$. asiaticus' in systematically collected leaves (collection 2). A total of 65 of the 72 (90.3\%) leaves that were systematically collected were asymptomatic, $5.5 \%$ were symptomatic, and $4.2 \%$ showed atypical symptoms including mineral deficiencies and other pathogens. Out of 65 asymptomatic leaves, $20 \%$ were ' $\mathrm{Ca}$. L. asiaticus' positive. All four symptomatic leaves were confirmed ' $\mathrm{Ca}$. L. asiaticus' positive, while ' $\mathrm{Ca}$. L. asiaticus' was detected in $33 \%$ of atypical symptomatic leaves (Table 1; Fig. 7).

Detection of ' $\mathrm{Ca}$. L. asiaticus' in the root samples. In total, 160 root samples were collected from HLB-infected 'Valencia' sweet orange trees on sour orange rootstock. The roots were differentiated between horizontally growing (137 samples) and vertically growing (23 samples) roots. From these samples, realtime PCR detected the presence of ' $\mathrm{Ca}$. L. asiaticus' in $132(96 \%)$ horizontal root and $18(78 \%)$ vertical root samples. Of the 160 root samples, 117 horizontal (89\% of the real-time PCR samples) and 18 vertical root samples (100\% of the real-time PCR samples) produced an expected 703-bp amplicon in the cPCR (Fig. 8). Subsequent analysis was based on real-time PCR results. These samples ranged in distance from the trunk between $0 \mathrm{~cm}$ (i.e., taken directly off the trunk) up to $377 \mathrm{~cm}$. A covariance model was fit to the data with response variable logarithm of the number of bacterial cells per gram of fresh weight and independent variables root type, distance, and

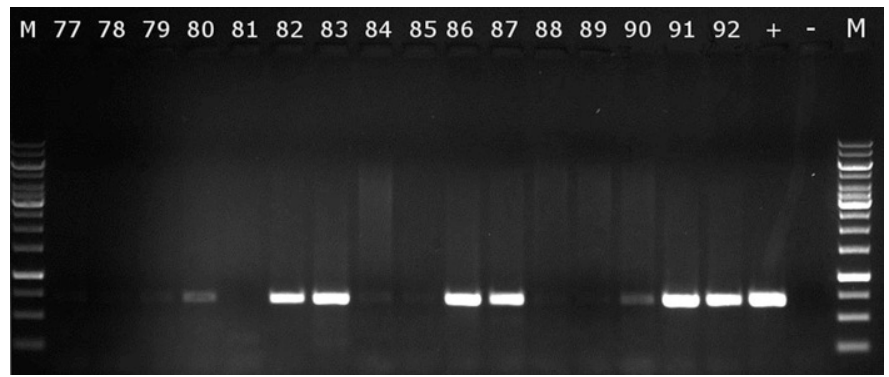

Fig. 8. The specific conventional polymerase chain reaction (PCR) amplification products with a size of $703 \mathrm{bp}$, obtained by using A2/J5 primers on the root DNA extract of Huanglongbing-asymptomatic sour orange rootstock grafted with 'Valencia' sweet orange. $\mathrm{M}=$ Thermo Scientific Gene Ruler 1-kb DNA ladder, lanes 77 to $92=$ root samples, $+=$ ' $C a$. L. asiaticus' PCR positive control DNA, and $-=$ nontemplate water control. tree number. Controlling for the individual tree, both root type and distance contributed to significant variation in ' $\mathrm{Ca}$. L. asiaticus' abundance $(P<0.0001)$. The model diagnostics (Table 2$)$ resulted in standard beta coefficients (a measure of the relative importance of the independent variables) of 0.26 for root type and -0.15 for distance. The variance inflation factor measures of correlation among independent variables were low. The residual analysis did not reveal any problems. Horizontal roots had higher abundances than vertical roots, and abundance decreased with distance from the trunk (Fig. 9).

Nucleotide sequence analyses. After removing primer and plasmid vector sequences, the 654-bp nucleotide sequences specific to ' $C a$. L. asiaticus' ribosomal protein genes of $\beta$ operon were obtained. A total of five sequences derived from 'Rio Red' leaf samples and eight sequences obtained from root samples of sour orange rootstock trees grafted with 'Valencia' sweet orange were sequenced. Overall, pairwise sequence identity averaged $99.4 \%$ (range: 98.3 to $100 \%$ ) with $99.5 \%$ identity among leaf samples, $99.2 \%$ among roots, and $99.5 \%$ between roots and leaves. Indeed, these sequences shared an average pairwise identity of $99.7 \%$ with 'Ca. L. asiaticus' strains gxpsy and psy62 (GenBank accession numbers CP004005 and CP001677, respectively), while individual pairwise comparisons ranged from 99.1 to $100 \%$ identical. Our sequences have been deposited in GenBank under accession numbers KR919749 to KR919752.

\section{DISCUSSION}

Our analysis supports the findings of Kunta et al. (2014b) that ' $\mathrm{Ca}$. L. asiaticus' is highly patchy within the canopy of infected trees. From the randomly collected leaf samples, we found a patchy distribution of ' $\mathrm{Ca}$. L. asiaticus' when the position of positive and negative leaves was plotted in the tree canopy. An example of one (second tree, the tree number counted from north to south) of the four trees is shown in Figure 7. It can be seen that there is no specific pattern of ' $\mathrm{Ca}$. L. asiaticus' distribution in the canopy and there are positive leaves among several negative leaves and vice versa. Indeed, even on symptomatic branches that consistently had much higher numbers of ' $\mathrm{Ca}$. L. asiaticus' bacteria than the asymptomatic branches (Fig. 1), 'Ca. L. asiaticus' was detectable in only $25 \%$ of leaves.

Despite the patchy distribution of ' $\mathrm{Ca}$. L. asiaticus' in the canopy, we were able to detect a directional bias in the abundance of ' $\mathrm{Ca}$. L. asiaticus'. Branches on the north side of trees housed significantly larger populations of ' $\mathrm{Ca}$. L. asiaticus' (Fig. 6B). In addition to the directional bias, there was spatial variation in the distribution of ' $C a$. L. asiaticus' within these directions. Branches to the south and to the west had higher abundance of ' $\mathrm{Ca}$. L. asiaticus' on leaves closer to the trunk (peaking at $75 \mathrm{~cm}$ ), while abundance peaked further from the trunk on northern branches (peaking at $150 \mathrm{~cm}$ ) (Fig. 6A). Young leaves, likely to provide nutrients needed for bacterial growth, tend to occur further from the trunk. Although young leaves in the interior of the canopy are shaded, it is well known that ACP are more attracted to the leaves exposed to light at the periphery. Exposed, these leaves are subject to a greater range of environmental fluctuation. As high temperatures have been shown to reduce ' $\mathrm{Ca}$. L. asiaticus' populations (Hoffman et al. 2013), it

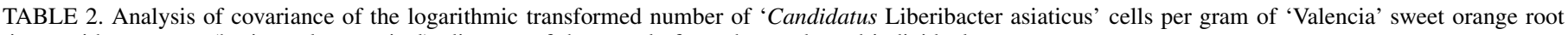
tissue with root type (horizontal or vertical), distance of the sample from the trunk, and individual tree

\begin{tabular}{|c|c|c|c|c|c|}
\hline Variable & Estimate & Standard error & Prob $>|\mathrm{t}|$ & Standard beta & Variance inflation factor \\
\hline Intercept & 7.135 & 0.0929 & $2.00 \mathrm{E}-117$ & 0 & - \\
\hline Tree \#10 & -0.335 & 0.0772 & $2.70 \mathrm{E}-05$ & -0.353 & 1.3444 \\
\hline Tree \#12 & -0.143 & 0.0861 & 0.0979 & -0.139 & 1.4165 \\
\hline Tree \#13 & 0.3591 & 0.0913 & 0.0001 & 0.3365 & 1.4821 \\
\hline Tree \#19 & 0.2482 & 0.0935 & 0.0088 & 0.228 & 1.4955 \\
\hline Root type $\mathrm{H}$ & 0.2421 & 0.0657 & 0.0003 & 0.2614 & 1.0208 \\
\hline Distance & -0.001 & 0.0006 & 0.033 & -0.154 & 1.0384 \\
\hline
\end{tabular}


is likely that the spatial patterns we observed are due to temperature differences mediated by solar radiation and wind direction (predominantly from the southeast with the branches on the northfacing side of the trees having a cooler microclimate more favorable to bacterial replication). Using the proportion of leaves in which we detected ' $\mathrm{Ca}$. L. asiaticus' among these positive trees, we can calculate the probability of a false-negative result using the pooled leaf diagnostic. By pooling 12 symptomatic leaves, the probability of a false negative result is $9.54 \times 10^{-8}$. However, a similar approach with asymptomatic leaves from infected trees raises that probability to 0.06 . Our results, however, suggest that further work into environmental influences on ' $\mathrm{Ca}$. L. asiaticus' distribution may lead to regionally or seasonally targeted sampling protocols that take advantage of these patterns to improve detection efficiencies.

To explore the distribution and abundance of ' $\mathrm{Ca}$. L. asiaticus' in the citrus root system, we uprooted five trees used in a previous study. In that previous study (Kunta et al. 2014b), five asymptomatic trees (R12-T8, R12-T10, R12-T12, R12-T13, and R12-T19) were surveyed for the presence of ' $\mathrm{Ca}$. L. asiaticus' in the canopy and in the roots. At that time, real-time PCR did not detect ' $\mathrm{Ca}$. L. asiaticus' in the canopy, but real-time PCR detected ' $C a$. L. asiaticus' in $23(58 \%)$ of the root samples from four trees (average $\mathrm{C}_{\mathrm{t}}: 31.43$; range: 26.22 to 36.38 ). One tree (R12-T19) showed no sign of ' $\mathrm{Ca}$. L. asiaticus' in any of the root or leaf samples.

Three months later, we uprooted those same trees for this study. By this time, tree R12-T19 showed evidence of ' $\mathrm{Ca}$. L. asiaticus' infection in the roots. Indeed, all five trees exhibited an even distribution of ' $\mathrm{Ca}$. L. asiaticus' throughout the root system. In fact, over the course of three months, the proportion of samples across all trees from which we could detect ' $\mathrm{Ca}$. L. asiaticus' grew from 58 to $96 \%$ of superficial fibrous roots. Despite this increase in distribution within the root system, there was still no evidence of ' $\mathrm{Ca}$. L. asiaticus' infection in the above-ground canopy, as all five trees remained asymptomatic.

Fibrous roots are both more abundant at superficial depths ( 0 to $30 \mathrm{~cm}$ deep) than among deeper vertical roots and more consistently infected with ' $\mathrm{Ca}$. L. asiaticus' than vertical roots. Specifically, the proportion of samples showing evidence of ' $\mathrm{Ca}$. L. asiaticus' in vertical roots (78\%) was significantly lower than in horizontal roots $(96 \%)$. This is perhaps not surprising in a flood irrigation system that stimulates superficial root growth resulting in increased phloem moving to these regions, perhaps carrying the bacteria to this tissue. Additionally, actively growing horizontal roots with nutrient-rich environment might favor the ' $\mathrm{Ca}$. L. asiaticus' colonization and multiplication compared with vertical roots. As in the canopy, there was significant variation in the spatial

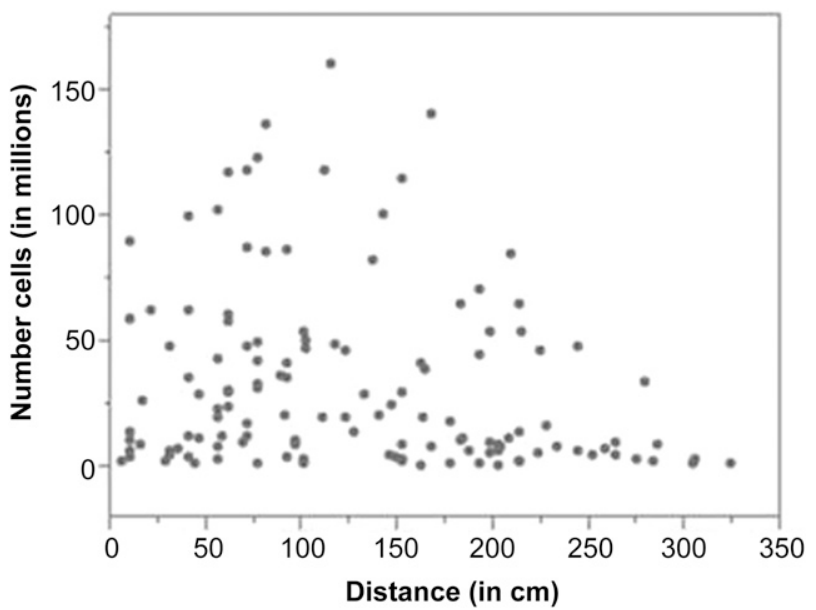

Fig. 9. The number (in millions) of 'Candidatus Liberibacter asiaticus' genome equivalents per gram of root tissue estimated in horizontal roots collected at different distances from trunk of 'Valencia' sweet orange trees. Each dot represents a positive detection. distribution of ' $\mathrm{Ca}$. L. asiaticus' among root tissue. In horizontal roots the greatest abundance and most consistent infections were found closer to the trunk (Fig. 9). Applying our observed 96\% detection rate to a tissue pooling strategy, 12 randomly collected fibrous roots would produce a probability of a false negative detection of $2 \times 10^{-17}$.

While leaf and root detection methods use different markers and are not quantitatively comparable, our results suggest that sampling from root tissue with the rpIL/rpIJ marker warrants further investigation to verify the detection method and assess if these patterns are widespread. It is expected that the proportion of tissue with detectable ' $\mathrm{Ca}$. L. asiaticus' is a function of the age of the infection and the number of inoculation sites. We detected ' $\mathrm{Ca}$. L. asiaticus' in $96 \%$ of horizontal root samples and predicted a falsenegative detection probability of $2 \times 10^{-17}$ with pooled samples. In addition to the consistency of detection, we found evidence that 'Ca. L. asiaticus' spreads rapidly among root tissue (from 58 to $96 \%$ coverage within three months), even in the absence of above-ground symptoms. Our DNA sequencing results show no evidence of false positives, as our sequences were identical or nearly identical to GenBank records for this species yet were clearly differentiated from closely related species (i.e., ' $\mathrm{Ca}$. L. africanus', ' $\mathrm{Ca}$. L. americanus'). However, the rpIL/rpIJ marker has not been subjected to thorough specificity testing using root samples. As the $16 \mathrm{~S}$ realtime PCR cross reacts with nontarget DNA in root DNA extracts, so too might the rpIL/rpIJ real-time PCR amplify nontarget sequences. Experiments designed to specifically test these questions are underway. Within the canopy, the symptomology of the tissue dramatically affects the probability of false negative detection. With symptomatic tissue, we estimate the probability of false negative detection to be quite low $\left(9.54 \times 10^{-8}\right)$. However in the absence of symptomatic tissue, the probability of false-negative detection can be as high as 0.06 . In light of this, our results indicating directional patterns in the abundance of ' $\mathrm{Ca}$. L. asiaticus' warrant further investigation. Our data suggests that, in Texas, the greatest opportunity for detection (highest titer and fewest negatives) of ' $\mathrm{Ca}$. L. asiaticus' in leaves was found on the ends of branches on the north side of the tree, perhaps as a result of environmental challenges. The findings from this study provide valuable information to the citrus industry on the distribution of ' $\mathrm{Ca}$. L. asiaticus' within trees that may aid development of sample collection methods for improved detection.

\section{ACKNOWLEDGMENTS}

This research was supported by a cooperative agreement 13-8130-0305CA between the United States Department of Agriculture (USDA)-Animal and Plant Health Inspection Service-Center for Plant Health Science and Technology and Texas Agricultural and Mechanical University at Kingsville. Mention of trade names or commercial products in this publication is solely for the purpose of providing specific information and does not imply recommendation or endorsement by the USDA, an equal opportunity employer. The forth author was partially supported by the NFSR at the MES of Bulgaria, grant number DFNI-I02/17, while on leave from the Institute of Mathematics and Informatics at the Bulgarian Academy of Sciences.

\section{LITERATURE CITED}

Altschul, S. F., Gish, W., Miller, W., Myers, E. W., and Lipman, D. J. 1990. Basic local alignment search tool. J. Mol. Biol. 215:403-410.

Bové, J. M. 2006. Huanglongbing: A destructive, newly-emerging, century-old disease of citrus. J. Plant Pathol. 88:7-37.

Capoor, S. P., Rao, D. G., and Viswanath, S. M. 1967. Diaphorina citri Kuwayama, a vector of the greening disease of citrus in India. Indian J. Agric. Sci. 37:572-576.

Coletta-Filho, M. L., Targon, P. N., Takita, M. A., De Negri, J. D., Pompeu, J., Jr., Machado, M. A., do Amaral, A. M., and Muller, G. W. 2004. First report of the causal agent of Huanglongbing ('Candidatus Liberibacter asiaticus') in Brazil. Plant Dis. 88:1382.

da Graça, J. V., Kunta, M., Sétamou, M., Rascoe, J., Li, W., Nakhla, M. K., Salas, B., and Bartels, D. W. 2015. Huanglongbing in Texas: Report on the first detections in commercial citrus. J. Citrus Pathol.iocv_journalcitruspathology_27939. 
Gottwald, T., Parnell, S., Taylor, E., Poole, K., Hodge, J., Ford, A., Therrien, L., Mayo, S., and Irey, M. 2008. Within-tree distribution of Candidatus Liberibacter asiaticus. Pages 270-273, in Proc. Int. Res. Conf. on Huanglongbing, Orlando, FL. Agricultural Research Service, Bethesda, MD.

Halbert, S. 2005. The discovery of huanglongbing in Florida. Page 50 in: Proc. 2nd Int. Workshop Citrus Canker and Huanglongbing. Florida Dept. Agric. Consumer Services, Div. Plant Industry, Gainesville, FL.

Hall, D. G., and Gottwald, T. R. 2011. Pest management practices aimed at curtailing citrus huanglongbing disease. Outlooks Pest Manage. 22:189-192.

Hocquellet, A., Toorawa, P., Bové, J. M., and Garnier, M. 1999. Detection and identification of the two Candidatus Liberobacter species associated with citrus huanglongbing by PCR amplification of ribosomal protein genes of the beta operon. Mol. Cell. Probes 13:373-379.

Hodges, A. W., and Spreen, T. H. 2012. Economic impacts of citrus greening (HLB) in Florida, 2006/07-2010/11. Food and Resource Economics Department, Florida Cooperative Extension Service, Institute of Food and Agricultural Sciences, University of Florida, Gainesville, FL.

Hoffman, M. T., Doud, M. S., Williams, L., Zhang, M.-Q., Ding, F., Stover, E., Hall, D., Zhang, S., Jones, L., Gooch, M., Fleites, L., Dixon, W., Gabriel, D., and Duan, Y.-P. 2013. Heat treatment eliminates 'Candidatus Liberibacter asiaticus' from infected Citrus trees under controlled conditions. Phytopathology 103:15-22.

Johnson, E. G., Wu, J., Bright, D. B., and Graham, J. H. 2014. Association of 'Candidatus Liberibacter asiaticus' root infection, but not phloem plugging with root loss on huanglongbing-affected trees prior to appearance of foliar symptoms. Plant Pathol. 63:290-298.
Kunta, M., da Graça, J. V., Malik, N., Louzada, E. S., and Sétamou, M. 2014a. Quantitative distribution of Candidatus Liberibacter asiaticus in the aerial parts of the HLB-infected citrus trees in Texas. HortScience 49:65-68.

Kunta, M., Viloria, Z., del Rio, H. S., and Louzada, E. S. 2014b. Diverse DNA extraction methods and PCR primers for detection of Huanglongbingassociated bacteria from roots of 'Valencia' sweet orange on sour orange rootstock. Sci. Hortic. (Amsterdam) 178:23-30.

Li, W., Hartung, J. S., and Levy, L. 2006. Quantitative real-time PCR for detection and identification of Candidatus Liberibacter species associated with citrus huanglongbing. J. Microbiol. Methods 66:104-115.

Li, W., Hartung, J. S., and Levy, L. 2007. Evaluation of DNA amplification methods for improved detection of "Candidatus Liberibacter species" associated with Citrus Huanglongbing. Plant Dis. 91:51-58.

Li, W., Levy, L., and Hartung, J. S. 2009. Quantitative distribution of 'Candidatus Liberibacter asiaticus' in citrus plants with citrus huanglongbing. Phytopathology 99:139-144.

Li, W., Li, D., Twieg, E., Hartung, J. S., and Levy, L. 2008. Optimized quantification of unculturable Candidatus Liberibacter spp. in host plants using real-time PCR. Plant Dis. 92:854-861.

Martinez, A. L., and Wallace, J. M. 1967. Citrus leaf-mottle-yellow disease in the Philippines and transmission of the causal virus by a psyllid, Diaphorina citri. Plant Dis. Rep. 51:692-695.

Wang, Z., Yin, Y., Hu, H., Yuan, Q., Peng, G., and Xia, Y. 2006. Development and application of molecular-based diagnosis for 'Candidatus Liberibacter asiaticus', the causal pathogen of citrus huanglongbing. Plant Pathol. 55: 630-638. 